\title{
Interaction of mesoscopic magnetic textures with superconductors
}

\author{
Serkan Erdin, ${ }^{1}$ Amin F. Kayali, ${ }^{1}$ Igor F. Lyuksyutov, ${ }^{1, *}$ and Valery L. Pokrovsky ${ }^{1,2}$ \\ ${ }^{1}$ Department of Physics, Texas A\&M University, College Station, Texas 77843-4242 \\ ${ }^{2}$ Landau Institute for Theoretical Physics, Moscow, Russia
}

(Received 20 November 2001; published 1 July 2002)

\begin{abstract}
Here we report a method to calculate the vortex and magnetization arrangement for a system of interacting superconductors and ferromagnets separated in space. The method is based on static London-Maxwell equations and the corresponding energy. Possible superconducting vortices are included in this system. Using this method we analyze screening currents in a superconducting film induced by magnetic textures in a thin magnetic film. We assume that the two films are parallel and positioned close to each to other, but interact exclusively via magnetic fields. We also consider possible vortices within this superconducting film and their interactions with magnetic texture. As an example of such magnetic texture we use a single magnetic dot with magnetization either perpendicular or parallel to the film. We derive a condition where spontaneous formation of one, two, or more vortices and antivortices is energetically favorable. We prove that, in the case of such a circular magnetic dot with perpendicular magnetization, when the vortex emerges in the superconducting film the normal component of magnetic field near the superconducting film changes sign outside of the dot range.
\end{abstract}

DOI: 10.1103/PhysRevB.66.014414

PACS number(s): 74.60.Ge, 74.25.Dw, 74.25.Ha, 74.76.-w

\section{INTRODUCTION}

Recent studies of mesoscopic heterogeneous ferromagnetic-superconducting systems (FSS's) opened an interesting class of physical effects. In FSS's the proximity effect which suppresses both order parameters can be avoided by introducing insulator oxide layers between ferromagnetic (FM) and superconducting (SC) components. Inhomogeneous magnetization of the magnetic texture generates a magnetic field penetrating into the superconductor. These fields induce superconducting currents. The magnetic field from these supercurrents interacts with the magnetic subsystem, providing strong interaction between the two subsystems. In order to study these effects several experimental groups fabricated periodic arrays of magnetic dots and antidots over or under a superconducting film. ${ }^{1-5}$ On the other hand several distinct mesoscopic FSS's were theoretically proposed and analyzed. Such systems include arrays of magnetic dots on the top of a SC film, ${ }^{6-8}$ FM-SC bilayers, ${ }^{9-11}$ magnetic nanorods embedded into a superconductor, ${ }^{12}$ magnetic stripes in superconducting films ${ }^{13}$ a layer of magnetic dipoles between two bulk superconductors, ${ }^{14}$ an array of magnetic dipoles mimicking the FM dots on SC film, ${ }^{15}$ and a domain wall in a thick magnetic film on the bulk superconductor. ${ }^{16,18}$ Earlier Marmorkos et al. ${ }^{17}$ theoretically considered a "giant" magnetic dot which generates several vortices in a bulk superconductor.

First experimental studies of FSS's focused on the pinning properties of magnetic dot arrays covered by a thin superconducting film ${ }^{1,2}$ resulting in the observation of the effect of the commensurability between an Abrikosov vortex lattice and the dot array on transport properties. ${ }^{1,2}$ However, this effect is not limited to the magnets interacting with superconductors, and was first found many years ago by Martinoli and his group. ${ }^{19}$ An investigation of the effects associated with the violation of time-reversal symmetry that are most specific for a FSS would hold greater promise. Theory predicts the occurrence of spontaneous currents in the ground state. ${ }^{10-13,20}$
Another such effect that was experimentally observed thus far is the asymmetry of the superconducting hysteresis in the presence of magnetic dots, reported by Morgan and Ketterson. ${ }^{3}$

In both theoretically proposed and experimentally realized FSS's the magnetic texture interacts with the SC current. Inhomogeneous magnetization generates a magnetic field outside the magnets, that in turn generates screening currents in superconductors which subsequently change the magnetic field. The problem must be solved self-consistently. Here we develop a method to calculate the inhomogeneous magnetization and supercurrents including SC vortices in the Londons approximation. We find elementary solutions for a circular magnetic dot on top of a SC film. London's approximation is sufficient, since the sizes of all the structures in the problem remarkably exceed the coherence length $\xi$. Our method reduces the solution to a search of proper positions for vortices at fixed magnetization. Conversely, if the magnetization is variable, it is necessary to find a distribution that minimizes the energy. The latter is presented as an integral over the volume occupied by the magnets and superconductors. In the next section we derive our method for the most general three-dimensional FSS. In Sec. III we apply this method to the case of very thin FM and SC films. In Sec. IV we consider magnetic dots on the top of SC film magnetized either perpendicular or parallel to the film.

\section{THREE-DIMENSIONAL SYSTEMS}

The total energy of a stationary FM-SC system reads

$$
H=\int\left[\frac{\mathbf{B}^{2}}{8 \pi}+\frac{m_{s} n_{s} \mathbf{v}_{s}^{2}}{2}-\mathbf{B} \cdot \mathbf{M}\right] d V,
$$

where $\mathbf{B}$ is the magnetic induction, $\mathbf{M}$ is the magnetization, $n_{s}$ is the density of SC electrons, $m_{s}$ is their effective mass and $\mathbf{v}_{s}$ is their velocity. We assume the SC density $n_{s}$ and the magnetization $\mathbf{M}$ to be separated in space. We also assume 
that the magnetic field $\mathbf{B}$ and its vector potential $\mathbf{A}$ asymptotically approaches zero at infinity. After the static Maxwell equations $\boldsymbol{\nabla} \times \mathbf{B}=(4 \pi / c) \mathbf{j}$ and $\mathbf{B}=\boldsymbol{\nabla} \times \mathbf{A}$ is an employed, the magnetic field energy can be transformed as follows:

$$
\int \frac{\mathbf{B}^{2}}{8 \pi} d V=\int \frac{\mathbf{j} \cdot \mathbf{A}}{2 c} d V .
$$

Although the vector potential enters explicitly into the last equation, it is gauge invariant due to the current conservation $\operatorname{div} \mathbf{j}=0$. When integrating by parts, we neglect the surface term. This approximation is correct if the field, vector potential, and current decrease sufficiently fast at infinity. The condition is satisfied in the simple systems analyzed in this study. The current $\mathbf{j}$ can be represented as a sum $\mathbf{j}=\mathbf{j}_{s}+\mathbf{j}_{m}$ of the SC and magnetic currents, respectively:

$$
\begin{gathered}
\mathbf{j}_{s}=\frac{n_{s} \hbar e}{2 m_{s}}\left(\boldsymbol{\nabla} \varphi-\frac{2 \pi}{\phi_{0}} \mathbf{A}\right), \\
\mathbf{j}_{m}=c \boldsymbol{\nabla} \times \mathbf{M} .
\end{gathered}
$$

We regard contributions from magnetic and SC currents into integral (2) separately, starting with the integral

$$
\frac{1}{2 c} \int \mathbf{j}_{m} \cdot \mathbf{A} d V=\frac{1}{2} \int(\boldsymbol{\nabla} \times \mathbf{M}) \cdot \mathbf{A} d V .
$$

Integrating by part and neglecting the surface term again, we arrive at the following result:

$$
\frac{1}{2 c} \int \mathbf{j}_{m} \cdot \mathbf{A} d V=\frac{1}{2} \int \mathbf{M} \cdot \mathbf{B} d V .
$$

We have omitted the integral over a remote surface $\oint(\mathbf{n}$ $\times \mathbf{M}) \cdot \mathbf{A} d S$. Such an omission is justified if the magnetization is confined to a limited volume. But for infinite magnetic systems it may be wrong even in simplest problems. This situation is discussed in Sec. II.

We then consider the contribution of the superconducting current $\mathbf{j}_{s}$ to integral (2). In the gauge-invariant equation (3), $\varphi$ is the phase of the SC carrier wave function and $\phi_{0}$ $=h c / 2 e$ is the (SC) flux quantum. Note that the phase gradient $\boldsymbol{\nabla} \varphi$ can be included into $\mathbf{A}$ as a gauge transformation. The exception are vortex lines, where $\varphi$ is singular. We use Eq. (3) to express vector potential $\mathbf{A}$ in terms of the supercurrent and the phase gradient:

$$
\mathbf{A}=\frac{\phi_{0}}{2 \pi} \boldsymbol{\nabla} \varphi-\frac{m_{s} c}{n_{s} e^{2}} \mathbf{j}_{s} .
$$

Plugging Eq. (7) into Eq. (2), we find

$$
\frac{1}{2 c} \int \mathbf{j}_{s} \cdot \mathbf{A} d V=\frac{\hbar}{4 e} \int \boldsymbol{\nabla} \varphi \cdot \mathbf{j}_{s} d V-\frac{m_{s}}{2 n_{s} e^{2}} \int j_{s}^{2} d V .
$$

Since $\mathbf{j}_{s}=e n_{s} \mathbf{v}_{s}$, the last term in this equation is equal to minus kinetic energy, and thus exactly compensates for the kinetic energy in the initial expression for the energy [Eq. (1)]. Collecting all the remaining terms, we obtain the following expression for the total energy:

$$
H=\int\left[\frac{n_{s} \hbar^{2}}{8 m_{s}}(\nabla \varphi)^{2}-\frac{n_{s} \hbar e}{4 m_{s} c} \nabla \varphi \cdot \mathbf{A}-\frac{\mathbf{B} \cdot \mathbf{M}}{2}\right] d V .
$$

This expression is correct with the caveat for a possible surface term for infinite magnetic systems. Note that integration in the expression for energy [Eq. (9)] proceeds over the volume occupied either by superconductors or by magnets. Equation (9) allows one to separate the energy of vortices, the energy of magnetization, and the energy of their interaction. Indeed, as we noted earlier, the phase gradient can be ascribed to the contribution of vortex lines alone. It can be represented as a sum of independent integrals over distinct vortex lines. The vector potential and the magnetic field can also be presented as a sum of magnetization-induced and vortex-induced parts $\mathbf{A}=\mathbf{A}_{m}+\mathbf{A}_{v}$, and $\mathbf{B}=\mathbf{B}_{m}+\mathbf{B}_{v}$, where $\mathbf{A}_{k}$, and $\mathbf{B}_{k}$ (the index $k$ is either $m$ or $v$ ) are determined as solutions of the Londons-Maxwell equations generated by magnetization or by vortices respectively. The effect of the SC screening of the magnetic field generated by magnetization is already included in the vector fields $\mathbf{A}_{m}$ and $\mathbf{B}_{m}$. If such a separation of fields is applied, the total energy [Eq. (9)] logically becomes a sum of terms containing vortex contributions alone, magnetic contributions alone and interaction terms. The purely magnetic component can be represented as a nonlocal quadratic form of the magnetization. The purely superconducting part becomes a nonlocal double integral over the vortex lines. Finally, the interaction term may be presented as a double integral over the vortex lines and the volume occupied by the magnetization that is bilinear in magnetization and vorticity. To avoid cumbersome formulas, we do not show these expressions explicitly.

Santos et al. ${ }^{14}$ developed a formalism for the calculation of magnetic fields and screening currents generated by a twodimensional array of magnetic dipoles confined between two bulk superconductors. This problem has a number of similarities with the one we consider. However, they did not consider any singular current distributions, i.e., vortices. The domain wall in a thick magnetic layer on a bulk superconductor was previously discussed by Bulaevsky and Chudnovsky. ${ }^{16}$ Their model is limited to screening effects only, while the generation of vortices is ignored. Helseth et al. ${ }^{18}$ theoretically analyzed the interaction between a vortex and domain wall in layers thicker than the domain wall width for the ferromagnetic layer, and thicker than the London penetration depth for the superconducting layer.

\section{TWO-DIMENSIONAL TEXTURES AND VORTICES}

Below we perform a detailed analysis in the case of parallel FM and SC films, with both films very thin and positioned close to each other. Neglecting their thickness, we assume both films to be located approximately at $z=0$. In some cases we need a higher level of accuracy. We then introduce a small distance $d$ between films, which in the end is assigned a zero value. Though the thickness of each film is assumed to be small, the two-dimensional densities of supercarriers $n_{s}^{(2)}=n_{s} d_{s}$ and magnetization $\mathbf{m}=\mathbf{M} d_{m}$ remain finite. Here $d_{s}$ is the thickness of the SC film and $d_{m}$ is the thickness of the FM film. The $3 d$ supercarrier density in the 
SC film is $n_{s}(\mathbf{R})=\delta(z) n_{s}^{(2)}(\mathbf{r})$ and the $3 \mathrm{~d}$ magnetization in the FM film is $\mathbf{M}(\mathbf{R})=\delta(z-d) \mathbf{m}(\mathbf{r})$, where $\mathbf{r}$ is the twodimensional radius vector and the $z$ direction is chosen to be perpendicular to the films. In what follows the $2 d$ SC density $n_{s}^{(2)}$ is assumed to be a constant and index (2) is omitted. Energy (9) for this special case takes the form

$$
H=\int\left[\frac{n_{s} \hbar^{2}}{8 m_{s}}(\boldsymbol{\nabla} \varphi)^{2}-\frac{n_{s} \hbar e}{4 m_{s} c} \boldsymbol{\nabla} \varphi \cdot \mathbf{a}-\frac{\mathbf{b} \cdot \mathbf{m}}{2}\right] d^{2} \mathbf{r},
$$

where $\mathbf{a}=\mathbf{A}(\mathbf{r}, z=0)$ and $\mathbf{b}=\mathbf{B}(\mathbf{r}, z=0)$. The vector potential satisfies the Maxwell-Londons equation

$$
\begin{aligned}
\boldsymbol{\nabla} \times(\boldsymbol{\nabla} \times \mathbf{A})= & -\frac{1}{\lambda} \mathbf{A} \delta(z)+\frac{2 \pi \hbar n_{s} e}{m_{s} c} \boldsymbol{\nabla} \varphi \delta(z) \\
& +4 \pi \boldsymbol{\nabla} \times[\mathbf{m} \delta(z)] .
\end{aligned}
$$

Here $\lambda=\lambda_{L}^{2} / d_{s}$ is the effective screening length for the SC film, and $\lambda_{L}$ is the London penetration depth. ${ }^{21}$

According to our general arguments, the term proportional to $\boldsymbol{\nabla} \varphi$ in Eq. (11) describes vortices. A plane vortex characterized by its vorticity $q$ and by position of its center on the plane $\mathbf{r}_{0}$, contributes a singular term to $\boldsymbol{\nabla} \varphi$,

$$
\boldsymbol{\nabla} \varphi_{0}\left(\mathbf{r}, \mathbf{r}_{0}\right)=q \frac{\hat{z} \times\left(\mathbf{r}-\mathbf{r}_{0}\right)}{\left|\mathbf{r}-\mathbf{r}_{0}\right|^{2}}
$$

and generates a standard vortex vector potential:

$\mathbf{A}_{v 0}\left(\mathbf{r}-\mathbf{r}_{0}, z\right)=\frac{q \phi_{0}}{2 \pi} \frac{\hat{z} \times\left(\mathbf{r}-\mathbf{r}_{0}\right)}{\left|\mathbf{r}-\mathbf{r}_{0}\right|} \times \int_{0}^{\infty} \frac{J_{1}\left(k\left|\mathbf{r}-\mathbf{r}_{0}\right|\right) e^{-k|z|}}{1+2 k \lambda} d k$.

Different vortices contribute independently into the vector potential and the magnetic field. In the limit of zero film thickness the usual Coulomb gauge $\operatorname{div} \mathbf{A}=0$ leads to a strong singularity in the vector potential. Therefore, it is reasonable to apply another gauge $A_{z}=0$. The calculations become simple in a Fourier representation. Following the prescriptions elaborated upon in Sec. II, we present the Fourier transform of the vector potential $\mathbf{A}_{\mathbf{k}}$ as a sum $\mathbf{A}_{\mathbf{k}}=\mathbf{A}_{m \mathbf{k}}+\mathbf{A}_{v \mathbf{k}}$ of independent contributions from magnetization and vortices. The equation for the magnetic part of the vector-potential reads

$$
\mathbf{k}\left(\mathbf{k} \cdot \mathbf{A}_{m \mathbf{k}}\right)-k^{2} \mathbf{A}_{m \mathbf{k}}=\frac{\mathbf{a}_{m \mathbf{q}}}{\lambda}-4 \pi i \mathbf{k} \times \mathbf{m}_{\mathbf{q}} e^{i k_{z} d},
$$

where $\mathbf{q}$ is the projection of the wave vector $\mathbf{k}$ onto the plane of the films: $\mathbf{k}=k_{z} \hat{z}+\mathbf{q}$. An arbitrary vector field $\mathbf{V}_{\mathbf{k}}$ in the wave-vector space can be fixed by its coordinates in a local frame of reference formed by the vectors $\hat{z}, \hat{q}, \hat{z} \times \hat{q}$ :

$$
\mathbf{V}_{\mathbf{k}}=V_{\mathbf{k}}^{z} \hat{z}+V_{\mathbf{k}}^{\|} \hat{q}+V_{\mathbf{k}}^{\perp}(\hat{z} \times \hat{q}) .
$$

Solutions of Eq. (14) are readily formulated in terms of these coordinates:

$$
\begin{gathered}
A_{m \mathbf{k}}^{\|}=-\frac{4 \pi i m_{\mathbf{q}}^{\perp}}{k_{z}} e^{i k_{z} d}, \\
A_{m \mathbf{k}}^{\perp}=-\frac{1}{\lambda k^{2}} a_{\mathbf{q}}^{\perp}+\frac{4 \pi i\left(k_{z} m_{\mathbf{q}}^{\|}-q m_{\mathbf{q} z}\right)}{k^{2}} e^{i k_{z} d} .
\end{gathered}
$$

Integration of the latter equation over $k_{z}$ allows one to find the perpendicular component of $\mathbf{a}_{\mathbf{q}}^{(m)}$ :

$$
a_{m \mathbf{q}}^{\perp}=-\frac{4 \pi \lambda q\left(m_{\mathbf{q}}^{\|}+i m_{\mathbf{q} z}\right)}{1+2 \lambda q} e^{-q d} .
$$

It follows from Eq. (14) that $a_{m q}^{\|}=0$. Note that Eq. (16) for the parallel component of the vector potential $A_{m \mathbf{k}}^{\|}$does not contain any information about the SC film. This component corresponds to a magnetic field equal to zero outside the FM film. Therefore, it is not essential for our problem.

The vortex part of the vector potential $\mathbf{A}_{v \mathbf{k}}$ also does not contain a $z$ component, since the supercurrents flow in the plane. The vortex-induced vector potential is

$$
\mathbf{A}_{v \mathbf{k}}=\frac{2 i \phi_{0}(\hat{q} \times \hat{z}) F(\mathbf{q})}{\mathbf{k}^{2}(1+2 \lambda q)},
$$

where $F(\mathbf{q})=\Sigma_{j} e^{i \mathbf{q} \cdot \mathbf{r}_{j}}$ is the vortex form factor, the index $j$ labels the vortices, and $\mathbf{r}_{j}$ are coordinates of the vortex centers. The Fourier transform of the vortex-induced vector potential at the surface of the SC film $\mathbf{a}_{v \mathbf{q}}$ reads

$$
\mathbf{a}_{v \mathbf{q}}=\frac{i \phi_{0}(\hat{q} \times \hat{z}) F(\mathbf{q})}{q(1+2 \lambda q)} .
$$

We express energy (10) in terms of the fields and vectorpotential Fourier transforms separating the purely magnetic, purely vortex, and interaction parts:

$$
H=H_{v}+H_{m}+H_{m v} .
$$

The vortex energy $H_{v}$ is the same as it would be in the absence of the FM film:

$$
H_{v}=\frac{n_{s} \hbar^{2}}{8 m_{s}} \int \boldsymbol{\nabla} \varphi_{-\mathbf{q}} \cdot\left(\nabla \varphi_{\mathbf{q}}-\frac{2 \pi}{\phi_{0}} \mathbf{a}_{v \mathbf{q}}\right) \frac{d^{2} q}{(2 \pi)^{2}} .
$$

However, the magnetic energy $H_{m}$,

$$
H_{m}=-\frac{1}{2} \int \mathbf{m}_{-\mathbf{q}} \cdot \mathbf{b}_{\mathbf{m q}} \frac{d^{2} q}{(2 \pi)^{2}},
$$

contains the screened magnetic field $\mathbf{b}$ and therefore differs from its value in the absence of the SC film, but does not depend on the vortex positions. The interaction energy reads

$$
\begin{aligned}
H_{m v}= & -\frac{n_{s} \hbar e}{4 m_{s} c} \int(\boldsymbol{\nabla} \varphi)_{-\mathbf{q}} \cdot \mathbf{a}_{m \mathbf{q}} \frac{d^{2} q}{(2 \pi)^{2}} \\
& -\frac{1}{2} \int \mathbf{m}_{-\mathbf{q}} \cdot \mathbf{b}_{v \mathbf{q}} \frac{d^{2} q}{(2 \pi)^{2}} .
\end{aligned}
$$

Note that only the form factor $F(\mathbf{q})$ bears information about the vortex arrangement. 
We illustrate how important the omitted surface term can be on the example of a homogeneous perpendicularly magnetized FM film interacting with a single vortex in the SC film. Lyuksyutov and Pokrovsky ${ }^{9}$ showed that the energy of this system is $\widetilde{\varepsilon}_{v}=\varepsilon_{v}-m \phi_{0}$, where $\varepsilon_{v}$ is the energy of the vortex in the absence of magnetic film, $m$ is the magnetization per unit area of the FM film and $\phi_{0}=h c / 2 e$ is the magnetic flux quantum. The term $-m \phi_{0}$ is the gain of energy of a magnetic fim in the magnetic field generated by the vortex. Let us analyze how this result is derived from the previously described general formalism. The vortex energy [Eq. (22)] is just equal to $\varepsilon_{v}$. The purely magnetic term [Eq. (23)] does not change in the presence of the vortex and is inessential. The first term in the interaction energy [Eq. (24)] is equal to zero since the infinite magnetic film does not generate a magnetic field outside itself. The second term of this energy is equal to $-m \phi_{0} / 2$. But this is only a half of the energy gain we discussed above. The second half of this gain is delivered by the surface term. Indeed, it is equal to

$$
\text { (1/2) } \begin{aligned}
\lim _{r \rightarrow \infty} \int_{0}^{2 \pi}(m \hat{r} \times \hat{z}) \cdot \mathbf{A} r d \varphi & =-(1 / 2) m \oint \mathbf{A} \cdot d \mathbf{r} \\
& =-m \phi_{0} / 2 .
\end{aligned}
$$

Erdin et al. argued that, after proliferation of vortices due to the instability, periodic stripe domains of opposite magnetization and vorticity occur in the bilayer. ${ }^{11}$ The periodic system is neutral on average. This means that the number of vortices in it is equal to the number of antivortices. Then the surface integral over two remote lines parallel to the stripes is proportional to the linear size of the system, since the magnetization and vector potential are periodic functions of coordinates and neither grows. Thus the surface integral can be neglected in comparison to the total energy obviously proportional to the film area. Thus the energy of a single vortex in the neutral system is $\widetilde{\varepsilon}_{v}=\varepsilon_{v}-m \phi_{0} / 2$.

\section{MAGNETIC DOTS}

In this section we consider the ground state of a SC film with a circular very thin FM dot grown upon it. The magnetization is assumed to be fixed, homogeneous inside the dot, and to be directed either perpendicular or parallel to the SC film. The problems we solve are (i) at what conditions vortices appear in the ground state, (ii) where they appear, and (iii) what the magnetic fields and currents in these states are. As in Sec. III, we assume the SC film to be very thin, plane, and infinite in lateral directions. Since the magnetization is confined within the finite dot, no integrals over infinitely remote surfaces or contours arise.

\section{A. Perpendicular magnetization}

Let both SC and FM films be infinitely thin, and place them at the heights $z=0$ and $z=d$, respectively. The SC film is infinite in lateral directions, while the FM film is finite and has a shape of regular circle with the radius $R$ (magnetic dot). The $2 d$ magnetization of the magnetic dot is $\mathbf{m}(\mathrm{r})$ $=m \hat{z} \sigma(R-r) \delta(z-d)$, where $\sigma(x)$ is a step function equal to +1 at positive arguments and 0 at negative ones. The vector potential and magnetic field induced by the dot in the presence of the SC film can be found by using Eqs. (17) and (18). The Fourier component of magnetization necessary for this calculation is

$$
\mathbf{m}_{\mathbf{k}}=\hat{z} \frac{2 \pi m R}{q} J_{1}(q R) e^{i k_{z} d},
$$

where $J_{1}(x)$ is the Bessel function. The Fourier transform of the vector potential reads:

$$
\begin{aligned}
A_{m \mathbf{k}}^{\perp}= & -\frac{i 8 \pi^{2} m R J_{1}(q R)}{k^{2}} \\
& \times\left(e^{-q d} \frac{2 q \lambda}{1+2 q \lambda}+\left(e^{i k_{z} d}-e^{-q d}\right)\right) .
\end{aligned}
$$

Though the difference in the round brackets in Eq. (26) looks to be always small (we recall that $d$ must be put zero in the final answer), we cannot neglect it since it implies a finite, not small, discontinuity in the parallel component of magnetic field at the two films faces. From Eq. (26) we immediately find the Fourier transforms of the magnetic field components:

$$
B_{m \mathbf{k}}^{z}=i q A_{m \mathbf{k}}^{\perp}, \quad B_{m \mathbf{k}}^{\perp}=-i k_{z} A_{m \mathbf{k}}^{\perp} .
$$

An important component of calculations is the Fourier transform of the vector potential at the superconductor surface:

$$
a_{m \mathbf{q}}^{\perp}=-\frac{i 8 \pi^{2} \lambda m R}{1+2 q \lambda} J_{1}(q R) .
$$

In the last equation we have replaced $e^{-q d}$ by 1 .

The inverse Fourier transformation of Eqs. (27) and (26) gives a magnetic field in real space,

$$
\begin{aligned}
B_{m}^{z}(\mathbf{r}, z) & =4 \pi \lambda m R \int_{0}^{\infty} \frac{J_{1}(q R) J_{0}(q r) e^{-q|z|}}{1+2 q \lambda} q^{2} d q, \\
B_{m}^{r}(\mathbf{r}, z)= & -2 \pi m R \int_{0}^{\infty} J_{1}(q R) J_{1}(q r) e^{-q|z|} \\
\times & {\left[\frac{2 q \lambda}{1+2 q \lambda} \operatorname{sgn}(z)+\operatorname{sgn}(z-d)-\operatorname{sgn}(z)\right] q d q, }
\end{aligned}
$$

where $\operatorname{sgn}(z)$ is the function equal to the sign of its $\operatorname{argu-}$ ment. Note that $B_{m}^{r}$ has discontinuities at $z=0$ and $z=d$ due to surface currents in the SC and FM films, respectively, whereas the normal component $B_{m}^{z}$ is continuous.

The symmetry arguments imply that a vortex, if it appears, must be located at the center of the dot. Indeed, for $R \gg \lambda$, an analytical calculation shows that the central position of the vortex provides a minimal energy. We have checked numerically that the central position is always energy favorable for one vortex. This fact is not trivial since the magnetic field of the dot is stronger near its boundary, and a 


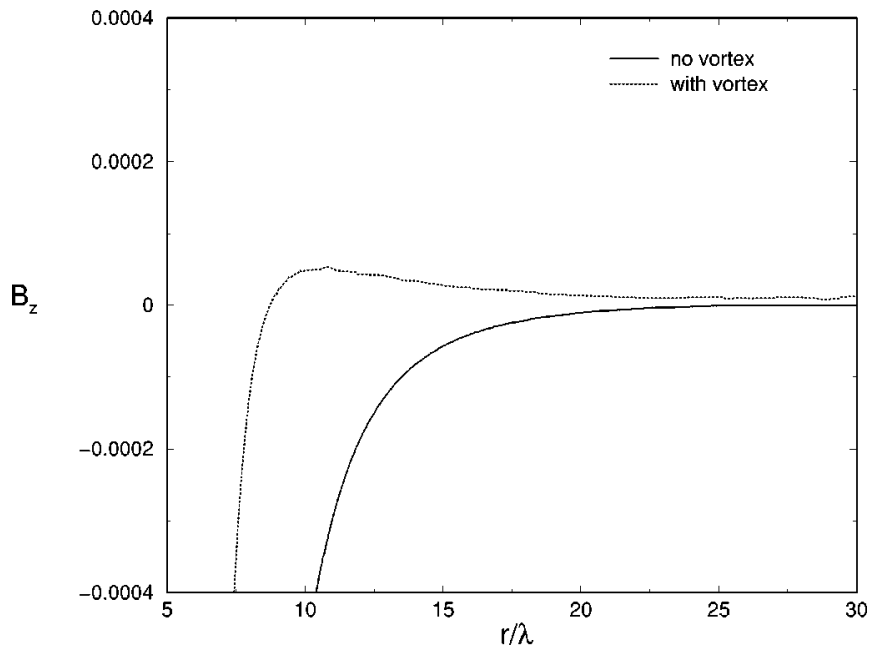

FIG. 1. Magnetic field of the dot with and without a vortex for $R / \lambda=5$ and $\phi_{0} / 8 \pi^{2} m R=0.05$.

violation of symmetry could be naively expected. However, the gain of energy due to interaction of the magnetic field generated by the vortex with magnetization decreases when the vortex approaches the boundary.

Another interesting problem is the sign of the perpendicular component of the magnetic field. The vector potential generated by a vortex is given by Eq. (19). The perpendicular component of magnetic field generated by the vortex is

$$
B_{v}^{z}=\frac{\phi_{0}}{2 \pi} \int_{0}^{\infty} \frac{J_{0}(q r) e^{-q|z|}}{1+2 \lambda q} q d q
$$

A numerical calculation based on Eqs. (29) and (31) shows that, in the presence of a vortex centered at $r=0, B_{z}$ on the SC film $(z=0)$ changes sign at some $r>R$ (see Fig. 1), but it is negative everywhere at $r>R$ in the absence of the vortex. The physical explanation for this fact is as follows. The dot itself is an ensemble of parallel magnetic dipoles. Each dipole generates a magnetic field, whose $z$ component on the plane passing through the dot has a sign opposite to that of the dipolar moment. However, the field exactly over and under the dipole has the same sign as the dipole and is strongly singular. The fields from different dipoles compete at $r<R$, but they have the same sign at $r>R$. The SC current tends to screen the magnetic field of the magnetization and to have an opposite sign. The field generated by a vortex at large distances decays slower than the screened dipolar field $\left(1 / r^{3}\right.$ vs $\left.1 / r^{5}\right)$. Thus the sign of $B_{z}$ is opposite to the magnetization at small values of $r$ (but larger than $R$ ) and positive at large $r$. The measurement of the magnetic field near the film may serve as a diagnostic tool to detect a SC vortex bound by a dot. To our knowledge, so far there has been no experimental measurement of this effect.

The energy of the system in the presence of a vortex can be calculated using Eqs. (21)-(24). The appearance of a vortex at the center of the dot changes the energy by the amount

$$
\Delta=\varepsilon_{v}+\varepsilon_{m v},
$$

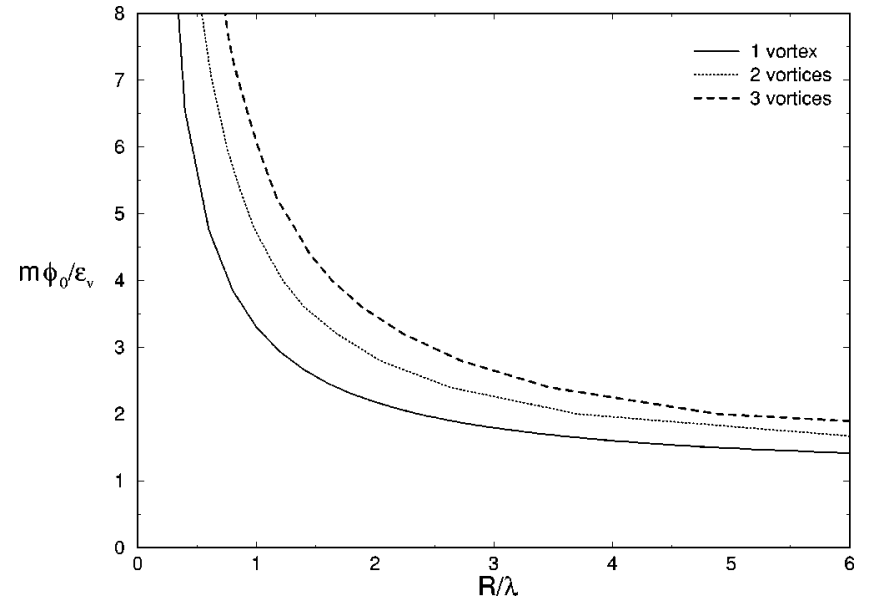

FIG. 2. Phase diagram of vortices induced by a magnetic dot. The lines correspond to the appearances of one, two, and three vortices, respectively.

where $\varepsilon_{v}=\varepsilon_{0} \ln (\lambda / \xi)$ is the energy of the vortex without magnetic dot, $\varepsilon_{0}=\phi_{0}^{2} /\left(16 \pi^{2} \lambda\right) ; \varepsilon_{m v}$ is the energy of interaction between the vortex and the magnetic dot given by Eq. (24). The direct substitution of the vector-potential, magnetic field and the phase gradient [see Eqs. (28) and (29)] into the equation for energy (24) leads to the following result:

$$
\varepsilon_{m v}=-m \phi_{0} R \int_{0}^{\infty} \frac{J_{1}(q R) d q}{1+2 \lambda q} .
$$

The vortex appears when $\Delta$ turns into zero. This criterion determines a curve in the plane of two dimensionless variables $R / \lambda$ and $m \phi_{0} / \varepsilon_{v}$. This critical curve separating regimes with and without vortices is depicted in Fig. 2. The asymptotic of $\varepsilon_{m v}$ for large and small values of $R / \lambda$ can be found analytically:

$$
\begin{gathered}
\varepsilon_{m v} \approx-m \phi_{0} \quad\left(\frac{R}{\lambda} \gg 1\right), \\
\varepsilon_{m v} \approx-m \phi_{0} \frac{R}{2 \lambda} \quad\left(\frac{R}{\lambda} \ll 1\right) .
\end{gathered}
$$

Thus, asymptotically, the curve $\Delta=0$ turns into a horizontal straight line $m \phi_{0} / \varepsilon_{v}=1$ at large $R / \lambda$ and logarithmically distorted hyperbola $\left(m \phi_{0} / \varepsilon_{v}\right)(R / \lambda)=2$ at small ratio $R / \lambda$.

Upon a further increase of either $m \phi_{0} / \varepsilon_{v}$ or $R / \lambda$, the second vortex becomes energy favorable. Due to symmetry, the centers of the two vortices are located on a straight line connecting the vortices with the center of the dot at equal distances from the center. The energy of the two-vortex configuration can be calculated by the same method. Curve 2 in Fig. 2 corresponds to this second phase transition. The occurrence of two vortices can be experimentally detected as the violation of circular symmetry of the field. In principle there exists an infinite series of such transitions. Here we limit ourselves to the first three, leaving a detailed analysis for a separate paper. The role of configurations with several vortices confined within the dot region and antivortices outside is not yet clear. 


\section{B. Parallel magnetization}

Next we consider an infinitely thin circular magnetic dot whose magnetization $\mathbf{M}$ is directed in the plane and is homogeneous inside the dot. An explicit analytical expression for $\mathbf{M}$ reads

$$
\mathbf{M}=m_{0} \theta(R-\rho) \delta(z) \hat{x},
$$

where $R$ is the radius of the dot, $m_{0}$ is the magnetization per unit area, and $\hat{x}$ is the unit vector along the $x$ axis. The Fourier transform of the magnetization is

$$
\mathbf{M}_{\mathbf{k}}=2 \pi m_{0} R \frac{J_{1}(q R)}{q} \hat{x} .
$$

The Fourier transform for the vector potential generated by the dot in the presence of a magnetic film takes the form

$$
\begin{aligned}
\mathbf{A}_{m \mathbf{k}}= & \hat{x} e^{i k d}\left[\frac{8 \pi^{2} m_{0} R}{k_{z}^{2}+q^{2}} J_{1}(q R) \cos \left(\phi_{q}\right)\right. \\
& \left.\times\left(\frac{i k_{z} e^{i k_{z} d}}{q}-\frac{e^{-q d}}{1+2 \lambda q}\right)\right] .
\end{aligned}
$$

Let a vortex-antivortex pair occur with the centers of the vortex and antivortex located at $x=+\rho_{0}$ and $x=-\rho_{0}$, respectively. Employing Eqs. (21)-(24) to calculate the energy, we find

$$
\begin{aligned}
E= & 2 \varepsilon_{0} \ln \left(\frac{\lambda}{\xi}\right)-4 \varepsilon_{0} \lambda \int_{0}^{\infty} \frac{J_{0}\left(2 q \rho_{0}\right)}{1+2 \lambda q} d q \\
& -2 m_{0} \phi_{0} R \int_{0}^{\infty} \frac{J_{1}(q R) J_{1}\left(q \rho_{0}\right)}{1+2 \lambda q} d q+E_{0},
\end{aligned}
$$

where $E_{0}$ is the dot self-energy. In a numerical analysis, we take $\lambda / \xi=100$.

Our numerical calculations indicate that the equilibrium value of $\rho_{0}$ is equal to $R$. The vortex-antivortex creation changes the energy of the system by

$$
\begin{aligned}
\Delta= & 2 \varepsilon_{0} \ln \left(\frac{\lambda}{\xi}\right)-4 \varepsilon_{0} \lambda \int_{0}^{\infty} \frac{J_{0}(2 q R)}{1+2 \lambda q} d q \\
& -2 m \phi_{0} R \int_{0}^{\infty} \frac{J_{1}(q R) J_{1}(q R)}{1+2 \lambda q} d q .
\end{aligned}
$$

The instability of the vortex-antivortex appearance develops when $\Delta$ changes sign. The critical curve $\Delta=0$ in the plane of dimensionless variables $m \phi_{0} / \varepsilon_{0}$ and $R / \lambda$ is plotted numerically in Fig. 3. In a region below this curve the creation of a vortex-antivortex pair is energy unfavorable, while in the region above the curve it is allowed. The phase diagram suggests that the smaller the radius $R$ of the dot, the larger the value of $m \phi_{0} / \varepsilon_{0}$ necessary to create the vortexantivortex pair. At large values of $R$ and $m \phi_{0} \geqslant \varepsilon_{0}$, the vortex is separated by a large distance from the antivortex. Therefore, their energy is approximately equal to that of two free vortices. This positive energy is compensated for by the at-

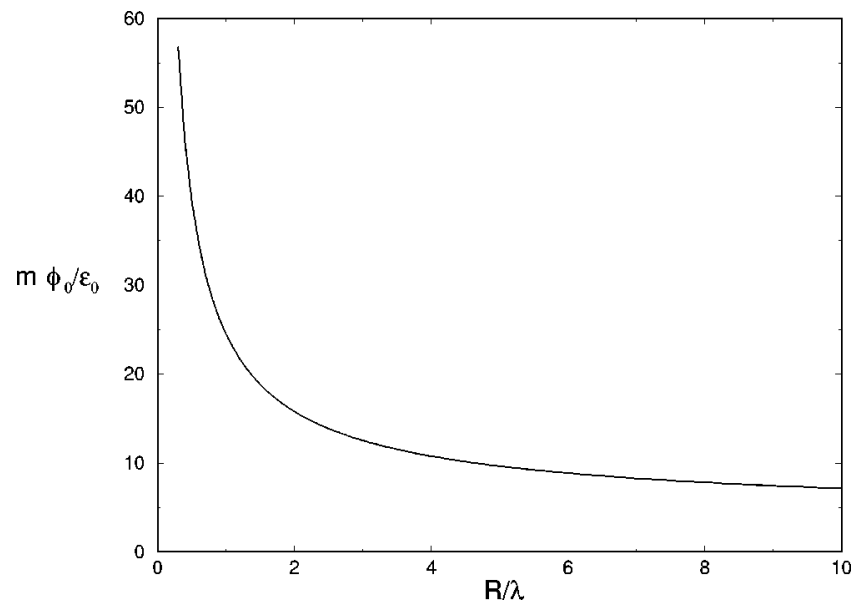

FIG. 3. Phase diagram for vortx-antivortx pair induced by the magnetic dot with in-plane magnetization.

traction of the vortex and antivortex to the magnetic dot. The critical values of $m \phi_{0} / \varepsilon_{0}$ seems to be numerically large even at $R / \lambda \sim 1$. This is a consequence of the comparatively ineffective interaction of in-plane magnetization with the vortex.

\section{CONCLUSION}

In conclusion, we presented a general formalism for the interaction between magnetic textures and superconductors in the London approximation. The problem is formulated as a variational principle. The variational functional (energy) is an integral over regions occupied either by a magnet or by a superconductor. It allows us to find positions of vortices and magnetization directly.

As applications, we have shown that vortices in superconducting films can be generated by magnetic dots normal to the film magnetization. We have found phase-transition curves separating the state without vortices from the state with one vortex, and the latter from the state with two vortices. In the case of one vortex under a dot we have shown that the perpendicular component of the magnetic field changes sign at some distance from the dot. This fact can be used for diagnostics of the vortex generation.

Superconducting vortices together with antivortices appear if the dot magnetization is parallel to the film. We have demonstrated that the magnetic dot size and its magnetization control the vortex generation and further transitions at which two or more vortices appear. The phase diagram reached for one dot implies even more complicated phase diagrams for arrays of dots.

\section{ACKNOWLEDGMENTS}

This work was supported by the NSF under Grant Nos. DMR 0103455 and DMR 0072115, and by the DOE under Grant No. DE-FG03-96ER45598. It is our pleasure to acknowledge discussions with D. G. Naugle, G. W. Crabtree, I. K. Schuller, and V. M. Vinokur. Our thanks are due to Dr. Olga Volpert for her valuable help. 
*Also at Institute of Physics, 252028 Kiev, Ukraine

${ }^{1}$ Y. Otani, B. Pannetier, J.P. Nozieres, and D. Givord, J. Magn. Magn. Mater. 126, 622 (1993); O. Geoffroy, D. Givord, Y. Otani, B. Pannetier, and F. Ossart, ibid. 121, 223 (1993).

${ }^{2}$ J.I. Martin, M. Velez, J. Nogues, and I.K. Schuller, Phys. Rev. Lett. 79, 1929 (1997).

${ }^{3}$ D.J. Morgan and J.B. Ketterson, Phys. Rev. Lett. 80, 3614 (1998).

${ }^{4}$ V.V. Metlushko et al., Solid State Commun. 91, 331 (1994); M. Baert et al., Phys. Rev. Lett. 74, 3269 (1995); V.V. Moschalkov et al., Phys. Rev. B 54, 7385 (1996); V.V. Metlushko et al., Europhys. Lett. 41, 333 (1998).

${ }^{5}$ M.J. Van Bael, J. Bekaert, K. Temst, L. Van Look, V.V. Moshchalkov, Y. Bruynseraede, G.D. Howells, A.N. Grigorenko, S.J. Bending, and G. Borghs, Phys. Rev. Lett. 86, 155 (2001); M. Lange, M.J. Van Bael, L. Van Look, K. Temst, J. Swerts, G. Guntherodt, V.V. Moshchalkov, and Y. Bruynseraede, Europhys. Lett. 51, 110 (2001); S.J. Bending, G.D. Howells, A.N. Grigorenko, M.J. Van Bael, J. Bekaert, K. Temst, L. Van Look, V.V. Moshchalkov, Y. Bruynseraede, G. Borghs, and R.G. Humphreys Physica C 332, 20 (2000).

${ }^{6}$ I.F. Lyuksyutov and V.L. Pokrovsky, Phys. Rev. Lett. 81, 2344 (1998)

${ }^{7}$ I. F. Lyuksyutov and V. L. Pokrovsky, in Superconducting Superlattices II: Native and Artificial, edited by Ivan Bozovic and Davor Pavuna, Proc. SPIE No. 3480 (SPIE, Bellingham, WA, 1998), pp. 230-235.

${ }^{8}$ D.E. Feldman, I.F. Lyuksyutov, V.L. Pokrovsky, and V.M. Vinokur, Europhys. Lett. 51, 110 (2000).
${ }^{9}$ I.F. Lyuksyutov and V.L. Pokrovsky, cond-mat/9903312 (unpublished); Mod. Phys. Lett. B 14, 409 (2000).

${ }^{10}$ I. F. Lyuksyutov, D. G. Naugle, and V. L. Pokrovsky, in Superconducting and Related Oxides: Physics and Nanoengineering $I V$, edited by Davor Pavuna and Ivan Bozovic, Proc. SPIE No. 4058 (SPIE, Bellingham, WA, 2000), pp. 376-387.

${ }^{11}$ S. Erdin, I.F. Lyuksyutov, V.L. Pokrovsky, and V.M. Vinokur, Phys. Rev. Lett. 88, 017001 (2002).

${ }^{12}$ I.F. Lyuksyutov and D.G. Naugle, Mod. Phys. Lett. B 13, 491 (1999).

${ }^{13}$ I.F. Lyuksyutov and D.G. Naugle, Physica C 341-348, 1267 (2000).

${ }^{14}$ J.E. Santos, E. Frey, and F. Schwabl, Phys. Rev. B 63, 054439 (2001).

${ }^{15}$ R. Sasik and T. Hwa, cond-mat/0003462 (unpublished).

${ }^{16}$ L.N. Bulaevskii and E.M. Chudnovsky, Phys. Rev. B 63, 012502 (2001).

${ }^{17}$ I.K. Marmorkos, A. Matulis, and F.M. Peeters, Phys. Rev. B 53, 2677 (1996)

${ }^{18}$ L.E. Helseth, P.E. Goa, H. Hauglin, M. Baziljevich, and T. Johansen, Phys. Rev. B 65, 132514 (2002).

${ }^{19}$ P. Martinoli, M. Nsabimana, G.A. Racine, H. Beck, and R. Clem, Helv. Phys. Acta 56, 765 (1983).

${ }^{20}$ I.F. Lyuksyutov and V.L. Pokrovsky, Mod. Phys. Lett. B 14, 409 (2000).

${ }^{21}$ A. A. Abrikosov, Introduction to the Theory of Metals (NorthHolland, Amsterdam, 1986). 